\title{
EVALUATION OF SIMULATED BONE LESION IN THE HEAD OF THE MANDIBLE BY USING MULTISLICE COMPUTED TOMOGRAPHY
}

\author{
Estevam Rubens UTUMI ${ }^{1}$, Andréia PERRELLA ${ }^{2}$, Marco Antonio Portela ALBUQUERQUE ${ }^{2}$, Carlos Alberto ADDE A $^{3}$
} Rodney Garcia ROCHA ${ }^{3}$, Marcelo Gusmão Paraíso CAVALCANTI ${ }^{4}$

1-DDS, Graduate Student, Department of Stomatology, Dental School, University of São Paulo, São Paulo, SP, Brazil.

2-DDS, MSc, PhD Student, Department of Stomatology, Dental School, University of São Paulo, São Paulo, SP, Brazil.

3-DDS, MSc, PhD, Professor of the Department of Stomatology, Dental School, University of São Paulo, São Paulo, SP, Brazil.

4- DDS,MSc, PhD, Professor of the Department of Stomatology, Dental School, University of São Paulo, São Paulo, SP, Brazil; Adjunct Professor,

Department of Radiology, College of Medicine, University of Iowa, Iowa, City, Iowa.

Corresponding address: Dr. Marcelo Cavalcanti - Avenida Prof. Lineu Prestes, 2227 - Disciplina de Radiologia - Departamento de Estomatologia FOUSP - 05508-900 - São Paulo, SP - e-mail: mgpcaval@usp.br

Received: August 7, 2008 - Modification: November 9, 2008 - Accepted: November 30, 2008

\begin{abstract}
$\mathcal{C}$

onventional radiography has shown limitation in acquiring image of the ATM region, thus, computed tomography (CT) scanning has been the best option to the present date for diagnosis, surgical planning and treatment of bone lesions, owing to its specific properties. Objective: The aim of the study was to evaluate images of simulated bone lesions at the head of the mandible by multislice CT. Material and methods: Spherical lesions were made with dental spherical drills (sizes 1, 3, and 6) and were evaluated by using multislice CT (64 rows), by two observers in two different occasions, deploying two protocols: axial, coronal, and sagittal images, and parasagittal images for pole visualization (anterior, lateral, posterior, medial and superior). Acquired images were then compared with those lesions in the dry mandible (gold standard) to evaluate the specificity and sensibility of both protocols. Statistical methods included: Kappa statistics, validity test and chi-square test. Results demonstrated the advantage of associating axial, coronal, and sagittal slices with parasagittal slices for lesion detection at the head of the mandible. Results: There was no statistically significant difference between the types of protocols regarding a particular localization of lesions at the poles. Conclusions: Protocols for the assessment of the head of the mandible were established to improve the visualization of alterations of each of the poles of the mandible's head. The anterior and posterior poles were better visualized in lateral-medial planes while lateral, medial and superior poles were better visualized in the anterior-posterior plane.
\end{abstract}

Key words: Tomography computed. Mandibular injuries. Radiography.

\section{INTRODUCTION}

Several CT studies regarding the temporomandibular joint (TMJ) have been recently published ${ }^{3,7,8,9,11,17}$. Conventional $\mathrm{x}$-rays have presented limitations for the evaluation of the condyle due to the overlapping of anatomical structures in its region and the distortion of images ${ }^{9}$. Computed tomography (CT) and magnetic resonance imaging (MRI) have been the methods of choice for evaluation of the TMJ structures ${ }^{5,6,9,12}$. In addition, CT has been the first option for diagnosis, surgical planning, and treatment of osseous trauma injuries due to their high specificity and sensitivity ${ }^{2,4,11,13}$.

It has been demonstrated that multislice detector $\mathrm{CT}$ (MDCT) is an accurate technique for evaluating bone changes in the condyle. MDCT produces very thin slices with high quality images in less acquisition time ${ }^{3,13}$.
This study evaluated CT images of simulated osseous lesions in the condyle in parasagittal slices and compared the validity of the images acquired using different MDCT protocols.

\section{MATERIAL AND METHODS}

Fifteen dry mandibles in which condyle lesions were produced involving only the cortical or cortical and medullar portions of the bone were evaluated. Spherical lesions were produced with high-speed and spherical drills sizes 1, 3, and 6 . The sites of the head of the mandible were classified by pole in: 1-anterior, 2-lateral, 3-posterior, 4-medial, and 5 -superior (Figure 1).

Induced lesions simulated sizes that were very similar to the diameter of a used dental burr $(1.0 \mathrm{~mm})$ and depth 


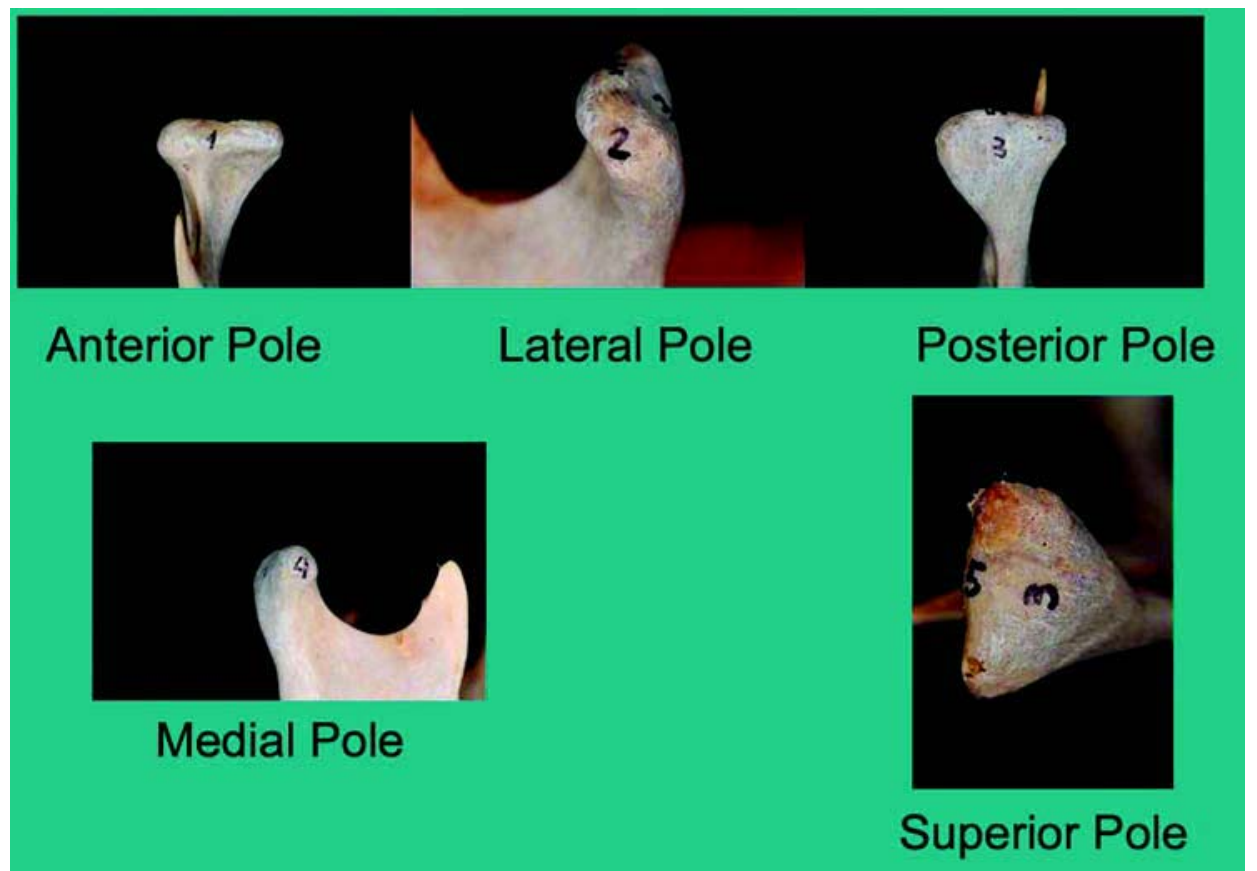

FIGURE 1- Classification of the head of the mandible by region

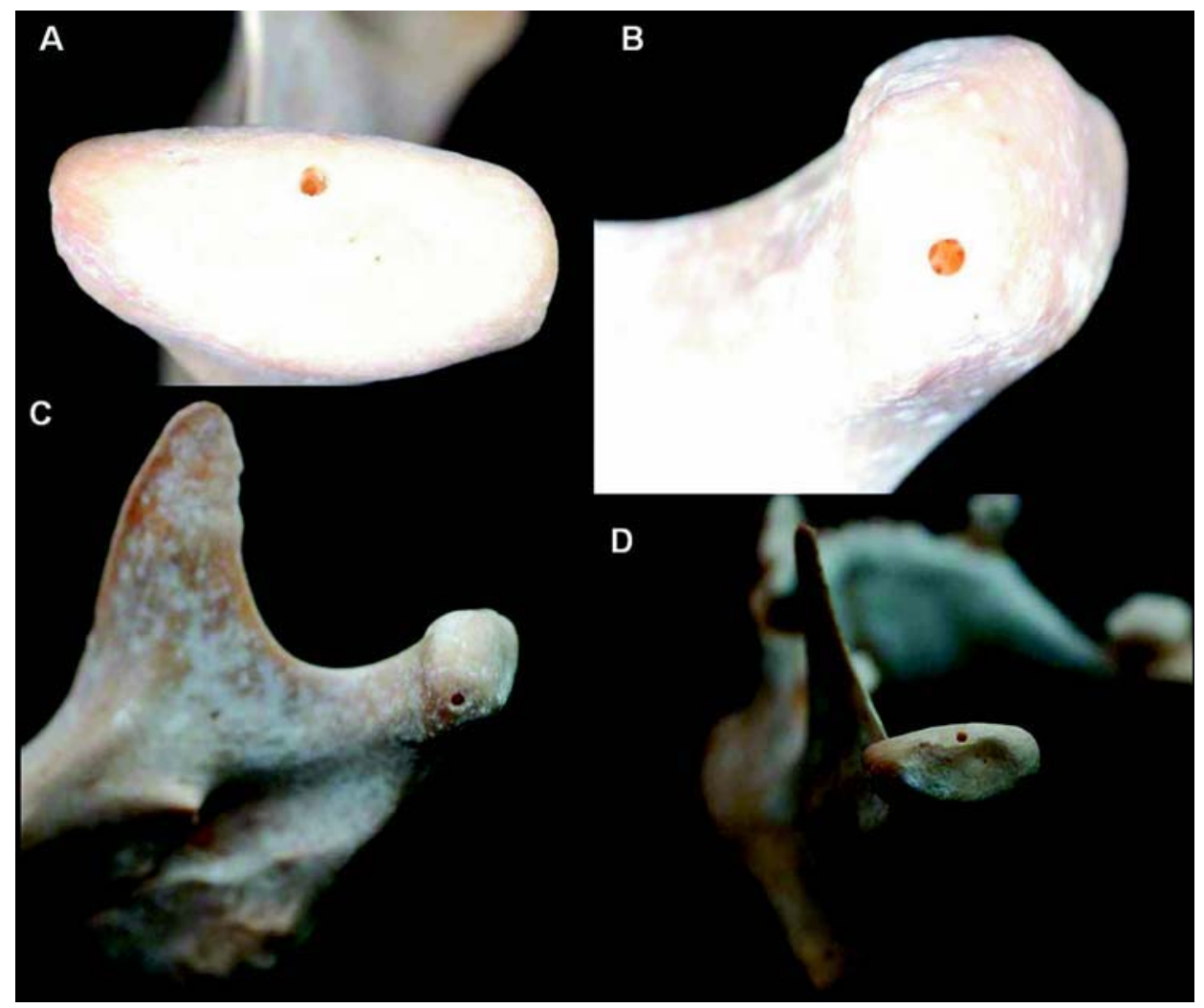

FIGURE 2- Views of the simulated lesions in different regions of the head of the mandible, (A) superior, (B and C) medial, (D) superior

equal to half the diameter of the burr (approximately 0.5 $\mathrm{mm}$ ) (Figure 2). Subsequently, mandibles underwent MDCT scans (64 rows, Aquilion, Toshiba Medical Inc., Tustin, CA, USA) with the following parameters: $0.5 \mathrm{~mm}$ of slice thickness, with $0.3 \mathrm{~mm}$ reconstruction interval by $0.5 \mathrm{~s}$, with
$120 \mathrm{kV}, 300 \mathrm{~mA}$ and matrix 1024x1024 using a bone tissue filter.

The original data were transferred to an independent Workstation (Dell Precision 420 hardware, Windows XP), using RadioStudio (version 1.7-Anne Solutions Company) 


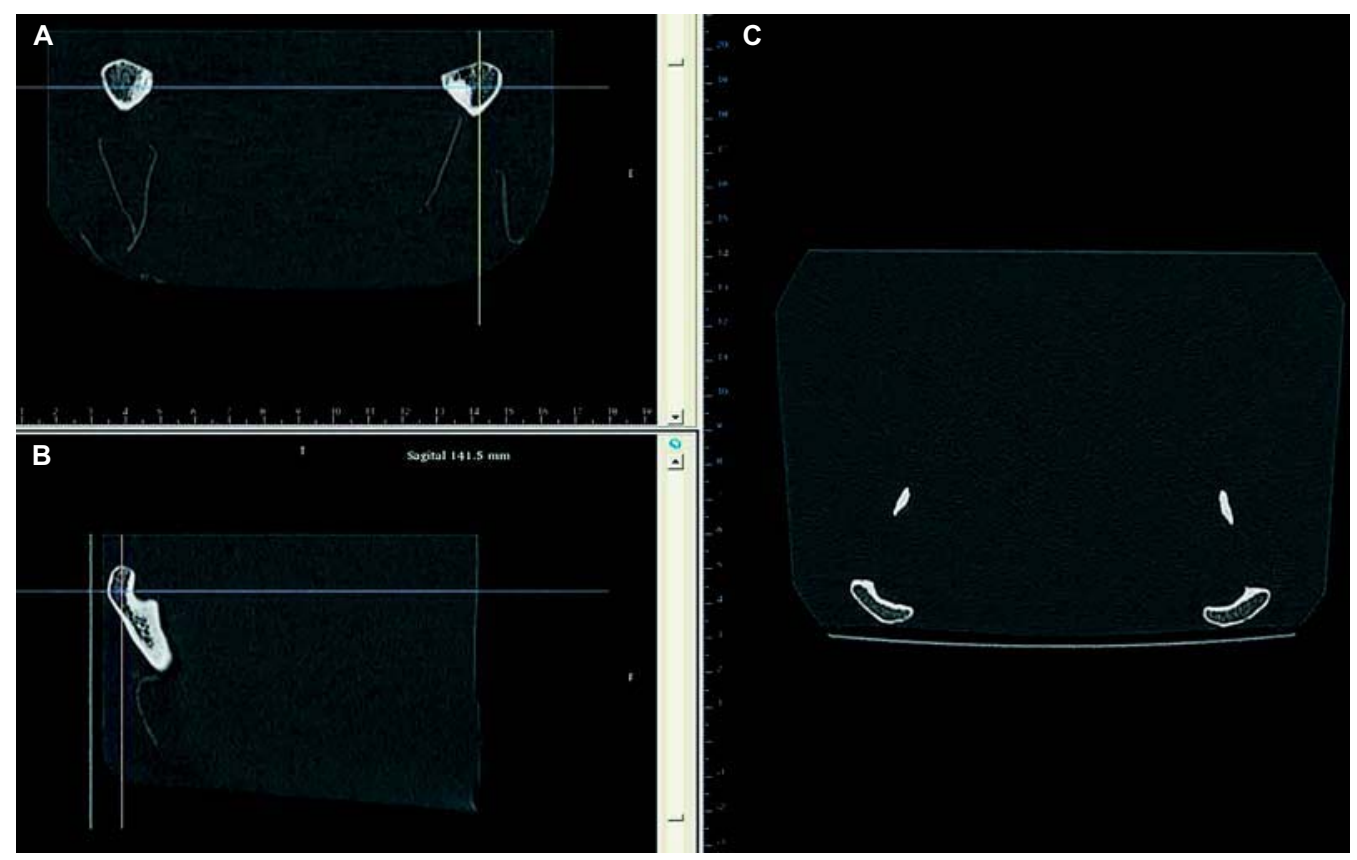

FIGURE 3- Multiplanar reconstructed (MPR) images: (A) axial, (B) coronal, and (C) sagittal, in bone window

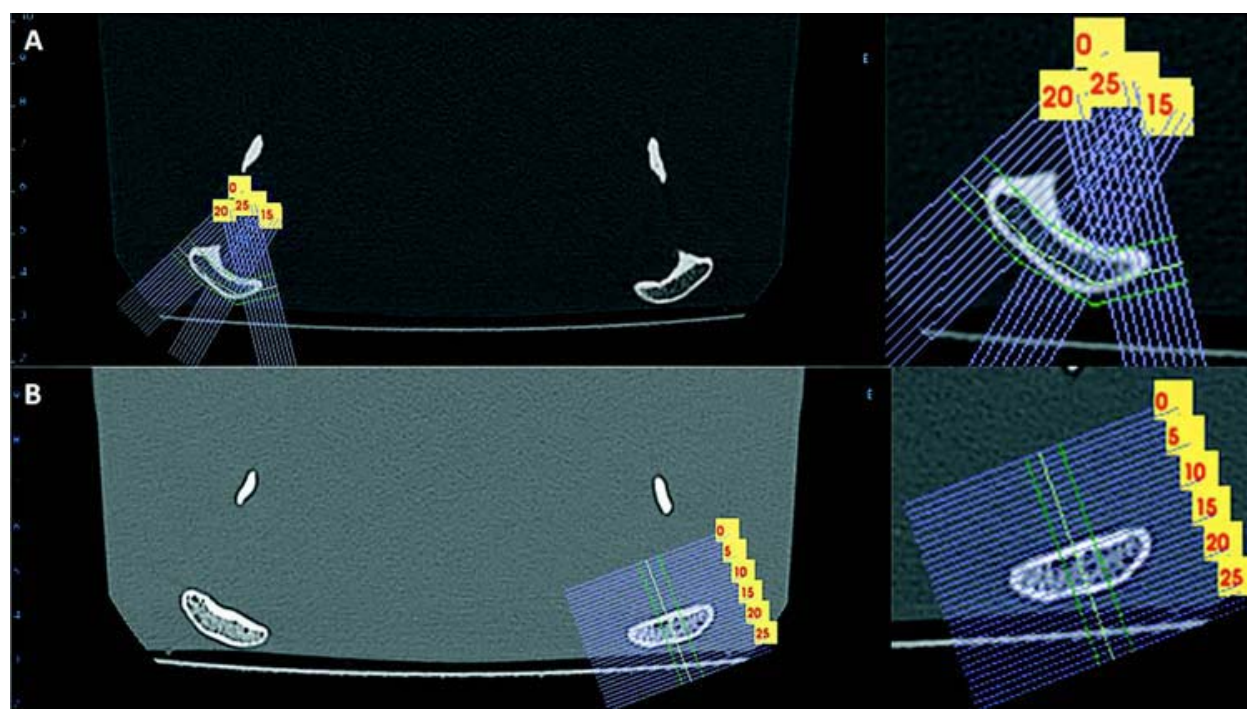

FIGURE 4- A- Parasagittal landmarks in the lateral-medial direction; B- Parasagittal landmarks in the anterior-posterior direction

to generate automatic and simultaneous multiplanar reconstructed images.

Analysis was performed using two protocols: (1) Multiplanar reconstruction (MPR): coronal, axial and sagittal images (Figure 3); (2) Parasagittal reconstructions (Figure 4A, 4B, and Figure 5). Axial images corresponded to original images and axial/MPR consisted of the association of axial and MPR images. Two previously calibrated observers interpreted the images with protocols in random order, in two different sessions, separated by an interval of, at least, 1 week. The observers evaluated the absence or the presence of bone destruction and its localization according to each protocol.

Statistical analyses included estimation of Kappa statistics, validity test (sensitivity-specificity) and chi-square test using SPSS software version 15.0 (Statistical Package will be Social Science, Chicago, IL, USA).

\section{RESULTS}

Results of intra- and inter- observer agreement are shown in Table 1. Observer 1, in the first measurement, had the highest agreement with the gold standard in the lateral polar region (0.75) and the lowest in the medial polar region (0.42). Observer 2 had the highest agreement with the gold standard in the lateral polar region (0.84) and the lowest in the medial polar region (0.57). Observer 1 , in the second measurement, presented the highest agreement with the gold standard in the superior polar region (0.90) and the lowest in the medial 
polar region (0.52).

Both observers had the lowest agreement with the goldstandard in the medial polar region of the axial, coronal and sagittal images.

In the analysis of parasagittal images (Table 2), observer 1 showed higher agreement with the gold standard in the superior polar region for parasagittal images for both first (0.79) and second (0.90) measurements. Observer 2 had higher agreement with the gold standard in anterior polar region (0.85). Observer 1 had the lowest agreement in medial $(0.37)$ and observer 2 in the posterior $(0.63)$ polar region.

In a total of 30 mandible heads evaluated and related to 5 poles, a total of 25 perforations was found in the medial pole (drill $1=10$, drill $3=7$, and drill $6=8$ ), 22 perforations in the lateral pole (drill $1=8$, drill $3=7$, and drill $6=7), 21$ perforations in the anterior pole $($ drill $1=7$, drill $3=7$, and
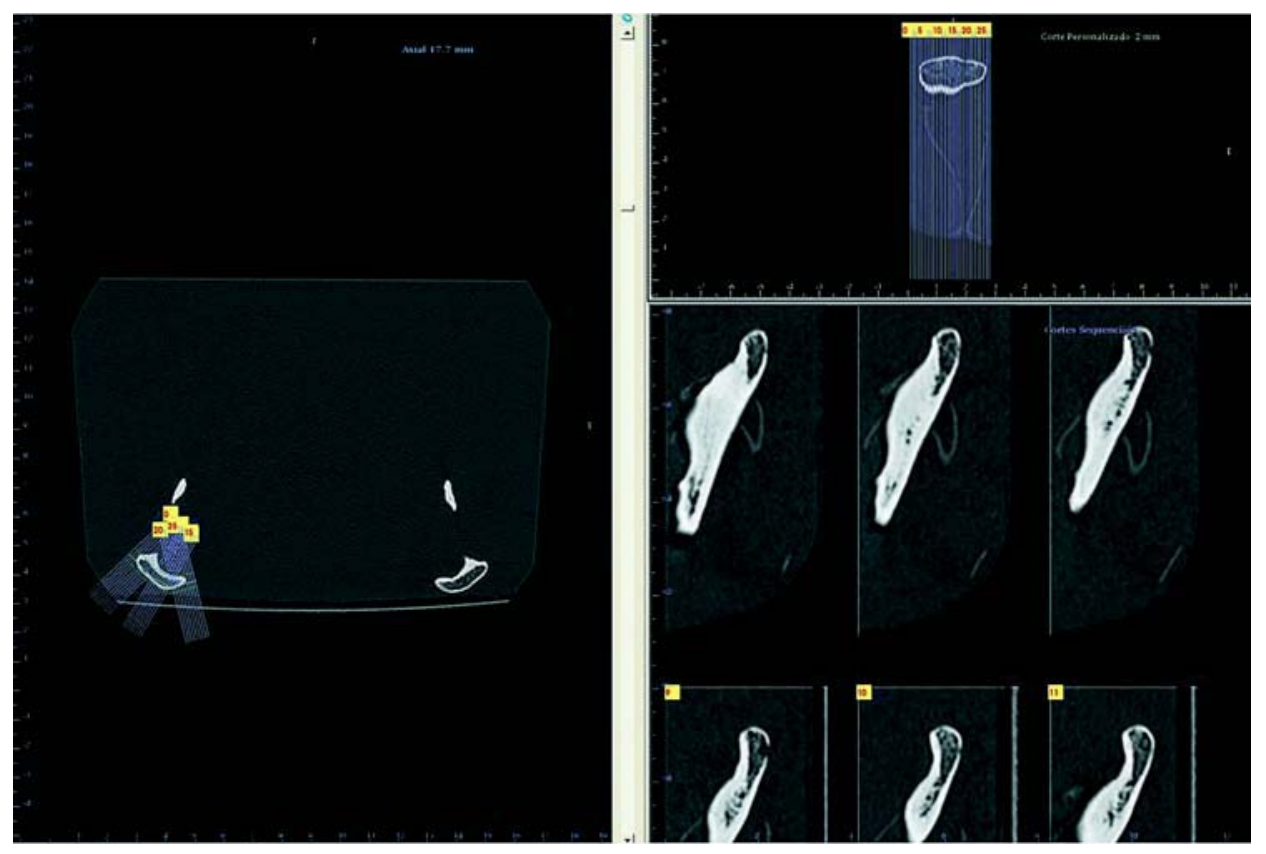

FIGURE 5- Parasagittal reconstructed images of the right side of the mandible

TABLE 1- Table of axial, coronal, sagittal images in comparison intra-observer, inter-observer with gold standard

\begin{tabular}{llllll}
\hline Kappa & Medial & Lateral & Anterior & Posterior & Superior \\
\hline Obs1 & 0.42 & 0.75 & 0.47 & 0.56 & 0.63 \\
p & 0.003 & $<0.001$ & 0.003 & 0.001 & $<0.001$ \\
Obs1' & 0.52 & 0.82 & 0.62 & 0.63 & 0.90 \\
p & 0.002 & $<0.001$ & $<0.001$ & $<0.001$ & $<0.001$ \\
Obs2 & 0.57 & 0.84 & 0.72 & 0.73 & 0.73 \\
p & $<0.001$ & $<0.001$ & $<0.001$ & $<0.001$ & $<0.001$ \\
\hline
\end{tabular}

Obs 1- First analysis of observer 1. Obs 1'- Second analysis of observer 1.

TABLE 2 - Table of parasagittal images in comparison intra-observer, inter-observer with gold standard

\begin{tabular}{llllll}
\hline Kappa & Medial & Lateral & Anterior & Posterior & Superior \\
\hline Obs1 & 0.37 & 0.75 & 0.66 & 0.53 & 0.79 \\
p & 0.004 & $<0,001$ & $<0,001$ & 0,001 & $<0,001$ \\
Obs1 & 0.64 & 0,73 & 0,77 & 0,81 & 0,90 \\
p & $<0.001$ & $<0.001$ & $<0.001$ & $<0.001$ & $<0.001$ \\
Obs2 & 0.79 & 0.84 & 0.85 & 0.63 & 0.67 \\
p & $<0.001$ & $<0.001$ & $<0.001$ & $<0.001$ & $<0.001$ \\
\hline
\end{tabular}

Obs 1- First analysis of observer 1. Obs 1'- Second analysis of observer 1. 
TABLE 3- Table of the values of sensibility and specificity

\begin{tabular}{|c|c|c|c|c|c|c|}
\hline Reconstruction & Sensibility & Medial & Lateral & Anterior & Posterior & Superior \\
\hline Axial, Coronal & Obs1 & 68.0 & 90.9 & 66.7 & 87.0 & 91.3 \\
\hline and Sagittal & Obs2 & 80.0 & 90.9 & 81.0 & 91.3 & 91,3 \\
\hline \multirow[t]{3}{*}{ Parasagittal } & Obs1 & 64.0 & 90.9 & 76.2 & 78.3 & 100,0 \\
\hline & Obs2 & 92.0 & 90.9 & 90.5 & 91.3 & 100.0 \\
\hline & Specificity & & & & & \\
\hline \multirow{2}{*}{$\begin{array}{l}\text { Axial, Coronal } \\
\text { and Sagittal }\end{array}$} & Obs1 & 100.0 & 87.5 & 88.9 & 71.4 & 71.4 \\
\hline & Obs2 & 100.0 & 100.0 & 100.0 & 85.7 & 85.7 \\
\hline \multirow[t]{2}{*}{ Parasagittal } & Obs1 & 100.0 & 87.5 & 100.0 & 85.7 & 71.4 \\
\hline & Obs2 & 100.0 & 100.0 & 100.0 & 71.4 & 57.1 \\
\hline
\end{tabular}

drill $6=7), 23$ perforations in the posterior pole $($ drill $1=6$, drill $3=9$, and drill $6=8$ ), 23 perforations in the superior pole (drill $1=7$, drill $3=8$, and drill $6=8$ ).

Sensitivity and specificity are shown in Table 3 . Highest sensitivity was observed for superior poles for both methods and observers (Table 3). Highest values for specificity were observed for medial, lateral, and anterior poles for both observers and methods.

\section{DISCUSSION}

Imaging of the TMJ region is limited with conventional radiography due to the overlap of anatomical structures, making visualization of the area of interest very difficult ${ }^{1,3,5,14,18}$. Multislice computed tomography represents an important advance in the field. Using this method, observers can reconstruct images with high resolution and less acquisition time ${ }^{3,13}$. Recent studies have demonstrated that $\mathrm{CT}$ is one of the best methods to evaluate abnormalities of the TMJ and adjacent structures, since it provides better visualization of soft and hard tissues without overlapping of images ${ }^{3,9,10,13,15,16,19}$. CT scanning has been the best option to the present date for the diagnosis of oral- and maxillofacial diseases, surgical planning and treatment of bone lesions ${ }^{9,15,19}$.

Ludlow, et al. ${ }^{12}$ (1995) published a comparative study in vitro for detection of bone alterations in the TMJ region. This study compared biplanar (sagittal and coronal) temporomandibular images produced by multidirectional tomographic and panoramic techniques. Series of dentin chips were placed at four locations on the head of the TMJ of a dried human skull. Tomographic and panoramic images were then taken. Biplanar tomography provided significantly more accurate assessment of condylar lesions than panoramic images $(\mathrm{p}=0.007)$. No statistically significant differences by location were found $(\mathrm{p}=0.592)$. The effects of observer and repeated observations were marginally significant at $\mathrm{p}=0.046$ and $\mathrm{p}=0.030$, respectively.
In comparison to the study of Ludlow, et al. ${ }^{12}$ (1995), the present investigation demonstrated statistically significant differences in relation to the position of the simulated lesions at the head of the mandible, influenced by the diameter of the drills and the number of perforations in each polar region. The types of images did not show significant differences regarding the percentages of agreement in polar regions. In the lateral, posterior and superior polar regions, the $\mathrm{p}$ value for the chi-square test was 1.0. In the medial and anterior polar regions, the $\mathrm{p}$ values were $\mathrm{p}=0.530$ and $\mathrm{p}=0.718$, respectively.

Warnke, et al. ${ }^{20}$ (1996) used dental scan software to reconstruct images. Those authors observed that the combination of axial CT with coronal and sagittal images provided more accurate images than conventional $\mathrm{CT}$. In their study, $100 \%$ of the pathological bone alterations were observed. Using software that could easily be installed in a personal computer, they showed that it is possible to reconstruct original images coming from any source with DICOM (Digital Imaging Communication in Medicine) images.

Perrella, et al. ${ }^{13}$ (2007) reported that values of sensitivity and specificity were $100 \%$ for both single-slice and multislice protocols for the detection of simulated lesion in the mandible's body. However, the acquisition method utilized limited the ability to detect the correct number of loci in multilocular lesions and the location of medullar invasion. The use of thinner slices when scanning a lesion was responsible for more effective results in the detection of medullar invasion and characterization of loci. In the present study, different values of sensitivity and specificity were found. The values were not influenced by the acquisition method used by Perrella, et al. (2007); they were influenced by the size of drill 1, which made the evaluation of different regions more difficult.

Cara, et al. ${ }^{3}$ (2007) evaluated and compared the validity of different single and multislice methods for analyses of simulated lesions in the head of the mandible. Sensitivity results were: axial single-slice method $1(62.7 \%)$, axial multi- 
slice method $2(66.2 \%)$, axial/multiplanar reconstruction single-slice protocol 3 (72.7\%), axial multi-slice/multiplanar reconstruction protocol 4 (93.1\%). Association of axial images with multiplanar reconstruction (MPR) using multislice CT scan demonstrated high accuracy in relation to single-slice method.

The present study determined the validity of images acquired by using multi-slice CT scans with different protocols in multiplanar reconstructions and parasagittal slices. Both observers presented greater sensitivity values in the superior polar region in both protocols. Specificity was higher for both observers in both protocols for medial, lateral, and anterior.

The association of CT protocols for visualization of the region of the head of the jaw was established in intention to improve the visualization of the presence of alterations of each region of the head of the jaw.

\section{CONCLUSION}

The validity of multislice CT was established for the detection of mandibular lesions using both protocols. Valid protocols are important to improve the visualization of structures of the mandibular polar region. Anterior and posterior poles were better visualized from a latero-medial perspective using parasagittal images. Lateral, medial, and the superior poles were better visualized from an anterior posterior perspective.

\section{ACKNOWLEDGEMENTS}

Dr. Estevam Rubens Utumi was supported by the São Paulo State Reserach Foundation, FAPESP (Grant \#2006/ 05328-0; Master's scholarship). Dr. Marcelo Cavalcanti was supported by the Brazilian The National Council for Scientific and Technological Development (Universal Project - grant \#484848/2006-2 and Research Productivity Scholarship - grant \#306509/2006-7). The authors would like to thank Dr. Flavio E. Hirai for reviewing the manuscript.

\section{REFERENCES}

1- Ahlqvist JB, Isberg AM. Validity of computed tomography in imaging thin walls of the temporal bone. Dentomaxillofac Radiol. 1999;28(1):139.

2- Cavalcanti MG, Vannier MW. Quantitative analysis of spiral computed tomography for craniofacial clinical applications. Dentomaxillofac Radiol. 1998;27(6):344-50

3- Cara AC, Gaia BF, Perrella A, Oliveira JX, Lopes PM, Cavalcanti MG Validity of single- and multislice CT for assessment of mandibular condyle lesions. Dentomaxillofac Radiol. 2007;36(1):24-7.

4- Costa e Silva AP, Antunes JL, Cavalcanti MG. Interpretation of mandibular condyle fractures using 2D- and 3D-computed tomography. Braz Dent J. 2003;14(3):203-8
5- Dixon DC. Diagnostic imaging of the temporomandibular joint. Dent Clin North Am. 1991;35(1):53-74.

6- Helms CA, Morrish RB Jr, Kircos LT, Katzberg RW, Dolwick MF. Computed tomography of the meniscus of the temporomandibular joint: preliminary observations. Radiology. 1982;145:719-22.

7- Hintze H, Wiese M, Wenzel A. Cone beam CT and conventional tomography for the detection of morphological temporomandibular joint changes. Dentomaxillofac Radiol. 2007;36(4):192-7.

8- Honey OB, Scarfe WC, Hilgers MJ, Klueber K, Silveira AM, Haskell $\mathrm{BS}$, et al. Accuracy of cone-beam computed tomography imaging of the temporomandibular joint: comparisons with panoramic radiology and linear tomography. Am J Orthod Dentofacial Orthop. 2007;132(4):42938 .

9- Hussain AM, Packota G, Major PW, Flores-Mir C. Role of different imaging modalities in assessment of temporomandibular joint erosions and osteophytes: a systematic review. Dentomaxillofac Radiol. 2008;37(2):63-71

10- Kijima N, Honda K, Kuroki Y, Sakabe J, Ejima K, Nakajima I. Relationship between patient characteristics, mandibular head morphology and thickness of the roof of the glenoid fossa in symptomatic temporomandibular joints. Dentomaxillofac Radiol. 2007;36(5):277-81.

11- Koyama J, Nishiyama H, Hayashi T. Follow-up study of condylar bony changes using helical computed tomography in patients with temporomandibular disorder. Dentomaxillofac Radiol. 2007;36(8):4727.

12- Ludlow JB, Davies KL, Tyndall DA. Temporomandibular joint imaging: a comparative study of diagnostic accuracy for the detection of bone change with biplanar multidirectional tomography and panoramic images. Oral Surg Oral Med Oral Pathol Oral Radiol Endod. 1995;80(6):735-43

13- Perrella A, Borsatti MA, Tortamano IP, Rocha RG, Cavalcanti MG. Validation of computed tomography protocols for simulated mandibular lesions: a comparison study. Braz Oral Res. 2007;21(2):165-9.

14- Pearson MH, Rönning O. Lesions of the mandibular condyle in juvenile chronic arthritis. Br J Orthod. 1996;23(1):49-56.

15- Sales MA, Oliveira JX, Cavalcanti MG. Computed tomography imaging findings of simultaneous bifid mandibular condyle and temporomandibular joint ankylosis: case report. Braz Dent J. 2007;18(1):74-7.

16- Santos DT, Cavalcanti MGP. Osteossarcoma of the temporomandibular joint. Report of two cases. Oral Surg Oral Med Oral Pathol Oral Radiol Endod. 2002;94:641-7.

17- Tsiklakis K, Syriopoulos K, Stamatakis HC. Radiographic examination of the temporomandibular joint using cone beam computed tomography. Dentomaxillofac Radiol. 2004;33(3):196-201.

18- Tsuruta A, Yamada K, Hanada K, Hosogai A, Tanaka R, Koyama J, et al. Thickness of the roof of the glenoid fossa and condylar bone change: a CT study. Dentomaxillofacial Radiol. 2003;32(4):217-21.

19- Utumi ER, Sales MAO, Shinohara EH, Takahashi A, Coracin FL, Rocha RG, et al. SAPHO syndrome with temporomandibular joint ankylosis: clinical, radiological,histopathological, and therapeutical correlations. Oral Surg Oral Med Oral Pathol Oral Radiol Endod. 2008;105(3):e67-e72.

20- Warnke T, Carls FR, Sailer HF. A new method for assessing the temporomandibular joint quantitatively by dental scan. J Craniomaxillofac Surg. 1996;24(3):168-72. 